\title{
WAVELET ANALYSIS CONSIDERATIONS FOR EXPERIMENTAL NONSTATIONARY FLOW PHENOMENA
}

\author{
M. L. S. Indrusiak ${ }^{a}$, \\ A. J. Kozakevicius ${ }^{b}$, \\ and S. V. Möller \\ ${ }^{a}$ Universidade do Vale do Rio dos Sinos \\ Av. Unisinos, 950; CEP 93022-000 \\ São Leopoldo, RS, Brazil \\ mlsperb@unisinos.br \\ ${ }^{\mathrm{b}}$ Universidade Federal de Santa Maria \\ Av. Roraima 1000; CEP 97105-900 \\ Santa Maria, RS, Brazi \\ ${ }^{\mathrm{c}}$ Universidade Federal do Rio Grande do Sul \\ Rua Sarmento Leite, 425; CEP 90050-170 \\ Porto Alegre, RS, Brazil \\ Received: December 12, 2015 \\ Revised: February 15, 2016 \\ Accepted: March 14, 2016
}

ABSTRACT

In this work, wavelet transforms are the analysis tools for studying transient and discontinuous phenomena associated to turbulent flows. The application in quest results from velocity measurements with hot wire anemometry in the transient wake considering a circular cylinder in an aerodynamic channel. Continuous and discrete wavelet transforms are applied and compared with the corresponding results given by the Fourier transform. For the continuous wavelet transform, the Morlet function was adopted as transform basis, and for the discrete case, the Daubechies orthonormal wavelet with 20 null moments. Results using the discrete wavelet packet transform are also presented and compared. A wake past a cylinder was analytically simulated and compared with the actual one, both in transient flow. The ability of the wavelet transforms in the analysis of unsteady phenomena and the potential of the wavelet approach as a complementary tool to the Fourier spectrum for the analysis of stationary phenomena is presented and discussed.

Keywords: transient flow, wavelet transforms, fourier transform

\section{NOMENCLATURE}

a scale coefficient

$b \quad$ position coefficient

C approximation set

$D \quad$ detail set

Fs sampling frequency, $\mathrm{Hz}$

$g_{n}$, wavelet filter

$h_{n} \quad$ wavelet filter

$j \quad$ dyadic scale parameter or level

$k \quad$ dyadic position parameter

$\mathrm{L}^{2}(\Re)$ space of square integrable functions

$m \quad$ wavelet packet coefficient

$N \quad$ degree of a polynomial function

$n \quad$ positive integer

$p \quad$ prime number

$\Re \quad$ real numbers set

$t$ time, $\mathrm{s}$

$x \quad$ time series

$\tilde{X} \quad$ wavelet transform

$\mathrm{Z} \quad$ integer numbers set

\section{Greek symbols}

$\psi \quad$ wavelet function

$\phi \quad$ scale function

\section{Subscripts}

c coarse

$$
\begin{array}{ll}
d & \text { detail } \\
J & \text { coarser index }
\end{array}
$$

\section{INTRODUCTION}

Experimental data of turbulence are usually presented as discrete time series representing continuous functions in time, such as velocity, temperature or pressure, sampled with a suitably chosen constant frequency, allowing recording and analysis by digital means. Analyses are usually characterized by statistical results such as averages and variances, correlation functions and spectral density functions, performed over a single "time history", since turbulence is considered a phenomenon of random background with the presence of coherent structures (Hussain, 1983) and the ergodicity hypothesis is usually accepted (Galanti and Tsinober, 2004). For the study of such phenomena, Fourier analysis has been a valuable and widely used analysis tool.

Back to the 30 's, pioneering experimental articles in turbulence already used hot wires, a technique employed even nowadays, when the first correlation measurements in a pipe flow were manually calculated (Taylor, 1936). It took more than thirty years until the calculations could be done with the help of computers. Comte-Bellot, 1965 and Lawn, 1971 used analogical band pass filters and true-rms voltmeters for spectral measurements in parallel plates, using hot wires, and pipe flow, using Laser 
Doppler Anemometry, respectively. Since then, the rapid development of informatics and computer-aided methods led to very fast data acquisition systems, computers and software, improving dramatically the analysis resources for experimental data.

The study of turbulence by means of Fourier analysis provides information on the frequencies involved and the interdependence of simultaneous phenomena, such as fluctuation of velocity and pressure in different positions (Hinze, 1975). Nevertheless, for nonstationary data, Fourier analysis is useless because the average values are not meaningful (Bendat and Piersol, 1993). Nonstationary data are the usual rule in field measurements; an extreme example is the case of typhoon winds (Huy et al., 2009). There are also transient flows generated by operating conditions (Indrusiak et al., 2005; Indrusiak and Möller, 2011) and other nonstationary phenomena like bistable flow which arise from stationary initial conditions (Zdravkovich and Stonebanks, 2000; Alam et al. 2003; Olinto et al., 2009). In transient and other nonstationary flows, besides the variable mean values, additional phenomena may appear, as the flow average velocity changes with time.

A discrete time series is considered nonstationary when its mean square values and autocorrelation functions are not invariant with time (Bendat and Piersol, 1986). According to these authors, the absence of a general methodology to analyze non-stationary random data is due partly to the fact that such a classification is a negative statement specifying only a lack of stationary properties, rather than a positive statement defining the precise nature of the nonstationarity. The consequence is the need of a specific model for each class of nonstationarity.

Literature shows the wavelet transform as a suitable tool for analyzing problems represented by nonstationary series and discontinuities. Since the last decade of the twentieth century, there have been studies that use the wavelet transform to understand the chaotic nature of turbulence in systems where the assumptions of ergodicity, homogeneity and isotropy are restricted (Meyer, 1993). The wavelet analysis has become widely used not only in turbulence studies, but also in various areas of knowledge, from music (e. g. Velarde et al., 2013) to image processing (e. g. Unser and Blu, 2003) and speaker recognition (e.g. Sumithra, 2014), from economics (e. g. Crowley, 2007) to medicine (e.g. Armstrong, 2011; Castillo et al., 2013).

However, the option for wavelet transform may not always contribute for further understanding of the analyzed data; in some situations Fourier analysis provides equivalent results. The same is true regarding the many possible choices of wavelet functions available and the proper selection of the wavelet transform can indeed become an issue (Farge, 1992).
The current work has therefore two main goals: to present a general overview of relevant aspects from the wavelet analysis and to bring them into perspective when applying this approach for analyzing nonstationary data, obtained from measurements with hot wire anemometry in laboratory flows. Continuous and discrete wavelet transforms were applied. For the former, the Morlet kernel is assumed, and for the latter, the orthonormal Daubechies Db 20 wavelet is applied.

\section{SIGNAL ANALYSIS}

Statistical signal analysis can be performed in time or frequency spaces. In time space, spectral information is not visible, and in frequency or Fourier space, time information is lost. In order to preserve time-frequency characteristics of signals, appropriate tools are continuous wavelet transforms and the analysis in wavelet space, whose time-scale domain allows an easy access to frequency information. Discrete wavelet transforms may be used as well, they usually are computed faster than the continuous transforms and the results can be presented as a set of frequency band time series of the same length of the original series.

\section{Fourier transform: some relevant remarks}

Analysis tools in time domain are mean value and central moments: variance, skewness and kurtosis as well as auto and cross correlations. In frequency domain, Fourier transform is extensively applied, both to the signal itself and to the probability density function. The latter originates energy (power or Fourier) spectrum, as stated by Einstein, 1914. A relevant aspect related to Fourier analysis is the Parseval identity for functions of finite energy establishing that total energy of the signal is preserved by Fourier transform (Körner, 1988). Power spectrum can also be estimated through finite Fourier transform of the original signal time history. A usual procedure used to enhance quality of spectrum of a stationary signal is to estimate the spectral values for each of a collection of sample records, acquired sequentially and average the results. A time window (e.g. Hanning) is applied to each sample record to taper the data to provide a more gradual entrance and exit and avoid anomalies in the estimated spectrum (Bendat and Piersol, 1993). Figure 1 explains graphically the procedure. A smoothing can also be done in frequency by averaging over a proper spectral bandwidth.

One of the drawbacks of applying Fourier transform is the incapability of recognizing the presence of bursts or transient phenomena that may or may not be observed in physical space, but will be completely hidden in the Fourier space, since the transform coefficients refer to the entire time domain of the signal. Fourier transform base components - 
sine and cosine functions - have infinite support i.e. they are defined for all $x$ in $\mathfrak{R}$ and are not identically zero in any finite interval contained in $\mathfrak{R}$, which means that they are located in frequency, but extend over the whole time domain. Therefore, Fourier coefficients represent an average for the entire time domain at each frequency considered.

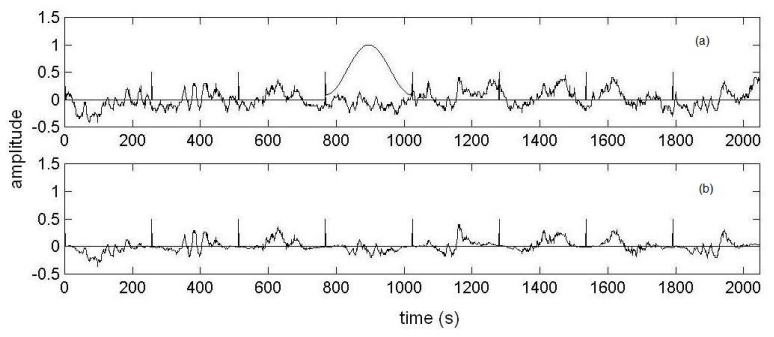

Figure 1. Schematic representation of the split of time series in blocks, multiplied by a spectral window (a). Result of applying the window to each block (b).

Windowed Fourier transform or Gabor transform was one of the attempts to allow time location, using Fourier transform (Gabor, 1946). The transform is done to the signal part within fixed sized windows applied to the signal. If the window is too narrow, low frequencies cannot be resolved. Conversely, by choosing a too large window, time localization of high frequencies will be lost.

\section{Wavelet transforms: an overview}

The continuous wavelet transform has emerged from the idea of compressing or expanding the window of windowed Fourier transform, keeping the same basis functions, compressed and dilated, allowing a better time location in high frequencies and better identification of low frequencies. To constitute a wavelet basis suitable for signal analysis applications, a function must be well located in both frequency and time. Due to this property of dual location, there is a balance in the resolutions in each area: the gain in temporal resolution is offset by a loss of resolution in frequency (Daubechies, 1992), according to the Heisenberg's uncertainty principle. This fact does not represent a limitation of the perception or of the analysis tools, but follows from the definition of frequency, which may not be measured instantaneously.

A wavelet, as the name suggests, is a "small" wave, which grows and decays in a limited and closed interval of time, being identically zero outside this region. This is exactly the so-called compact support property, satisfied by some of the possible wavelet families, as the orthonormal Daubechies wavelets. A "large" wave, by contrast, would be the sine/cosine functions for example, which keep oscillating all over the real domain (Percival and Walden, 2000). In other words, the basis created by dilations and translations of wavelet functions are transient, and therefore are more suitable for analyzing transient functions. There are many functions with the required admissibility property, which imposes a finite energy Fourier transform of the candidate function, but not all of them form an orthogonal basis for the space of the square integrable functions, $\mathrm{L}^{2}(\mathfrak{R})$.

Parseval identity applies also to wavelet transforms, ensuring that the energy of the signal is preserved by the transforms.

\section{Continuous wavelet transform}

The standard formulation for the wavelet function $\psi(t)$ that generates the continuous wavelet transform (CWT) is given, depending on its dilations and translations, as follows:

$$
\psi_{a, b}(t)=\frac{1}{\sqrt{a}} \psi\left(\frac{t-b}{a}\right) \quad a, b \in \mathfrak{R}, \quad a>0
$$

where $a$ and $b$ are respectively the scale and position coefficients. The continuity of the transform is due to the continuous variation of coefficients $a$ and $b$. This means that even in the analysis of a discrete signal, this analysis will be performed on all possible scales and at every point of the domain. These bases are not orthogonal, since the information is repeated in more than one coefficient, and therefore generates a large amount of redundant data.

The wavelet transform, therefore, is the application which associates a signal $x(t)$ to its wavelet coefficients, given by:

$$
\widetilde{X}(a, b)=\int_{-\infty}^{\infty} x(t) \psi_{a, b}(t) d t
$$

\section{Discrete wavelet transforms}

Discrete wavelet transform could simply be thought as a restriction of continuous version, when coefficients $a$ and $b$ no longer vary continuously, but assume dyadic (or any other $p$-adic, with $p$ prime) values. However, discrete wavelet function families and their transforms are themselves another entire area of research, especially because of their direct connection with filter banks, (Strang and Nguyen, 1996), and the fast formulations for their transforms (Unser and Blu, 2003, Mallat, 1989).

Discrete wavelet families are defined by a compact supported scaling function $\phi(t)$, an associated wavelet function $\psi(t)$ (also with compact support), and again their translations and dilations, assuming $j$ as dyadic scale parameter and a finite amount of possible $k$ values within each scale. Nevertheless, just some of these functions are defined analytically (e. g. Haar wavelet), and the main property characterizing these families is their 
association to finite length coefficient vectors, called wavelet filters $h_{n}$ and $g_{n}$, which allow the computation of these function values for any value $t$ in the real line $\mathfrak{R}$, as well as the fast discrete transforms.

The discrete wavelet transform (DWT) is thus given by:

$$
\tilde{X}_{d}(j, k)=\sum_{k} x(t) \Psi_{j, k}(t) \quad j, k \in \mathrm{Z}
$$

For each level $j$ the part of the signal containing the mean values (lower frequencies) is given by (Mallat, 1998):

$$
\tilde{X}_{c}(j, k)=\sum_{k} x(t) \phi_{j, k}(t)
$$

The DWT of a series with $2^{n}$ elements is computed for $1 \leq j \leq J$, being $J \leq n$ a convenient arbitrary choice for the coarser desired level. Then, any discrete time series with a sampling frequency $F s$ can be represented by:

$$
x(t)=\sum_{k} \tilde{X}_{c}(J, k) \phi_{J, k}(t)+\sum_{j \leq J} \sum_{k} \widetilde{X}_{d}(j, k) \psi_{j, k}(t)
$$

where the first term is the approximation $\mathrm{C}_{J}$ of the signal at scale $J$, which corresponds to frequency interval $\left[0, F_{S} / 2^{J+1}\right]$ and the inner summation of the second term are details $D_{j}$ of the signal at scales $\mathrm{j}$ $(1 \leq \mathrm{j} \leq \mathrm{J})$, which corresponds to frequency intervals $\left[F s / 2^{j+1}, F s / 2^{j}\right]$. Indeed, the DWT has the ability to split the whole range of signal frequencies associated with different scales of resolution and different characteristics in a manner similar to a bank of analog filters.

Again, the wavelet functions considered for discrete transforms form a basis in the space of integrable functions $\mathrm{L}^{2}(\mathfrak{R})$, since they generate, scale by scale, families of discrete nested spaces that, in the limit, cover the entire space $\mathrm{L}^{2}(\mathfrak{R})$, satisfying the concept of multiresolution analysis (Mallat, 1989, Daubechies, 1992). The different types of available bases can be classified with respect to symmetry or orthonormality of the functions $\phi(t)$ and $\psi(t)$ (and their translations and dilations), as well as with respect to the smoothness of these functions. In this sense, the smoothness is translated as null moment property, which states that polynomial functions until a certain degree $N$ can be represented exactly by scaling functions, having null projections with respect to the associated wavelets at the same scale. This property is directly related to the size of the wavelet filters, $h_{n}$ and $g_{n}$, which are responsible for the signal decomposition in the scaling and in the wavelet function directions, respectively. In the case of wavelets from the orthonormal Daubechies family, for example, the size is then $2 N$. The functions of this family are many times labeled as $\mathrm{DbN}$, indicating the number of null moments considered.

Fast wavelet transform applied to a time series given in a resolution level $j$ can then be interpreted as the convolution of this series, given by an input vector $x$ with its $2^{j}$ discrete values, with the wavelet filters $h_{n}$ and $g_{n}$, that define the scaling function and wavelet function. Therefore, the initial vector $x$ (or $C_{0}$ ) and, subsequently, each vector $C_{j}$ are then split in two other half sized vectors, $C_{j+1}$ and $D_{j+1}$, where $j+1$ indicates the next coarser level obtained by the decomposition. In Figure 2(a) a representation for the decimated fast DWT is shown, whose pyramidal algorithm is due to Mallat (1989). In the undecimated version of the transform, both vectors $C_{j+1}$ and $D_{j+1}$ keep the same size as the original vector $x$ at each one of the decomposition levels of the transform.

A modification of the pyramidal algorithm yields the so called discrete wavelet packet transform (DWPT), where each detail series is wavelet transformed in two series, with respectively lower and upper half bandwidth frequency interval (Meyer, 1993). For each level $j$ one can obtain $2^{j}$ successive intervals of equal bandwidth. This recursive transformation of detail series is represented in a binary tree, Fig. 2(b). Any admissible binary tree can be chosen for representation of the signal in the wavelet space, with frequency intervals of variable bandwidths, according to the analysis to be done. An admissible tree is any binary tree where each node has either 0 or 2 branches.

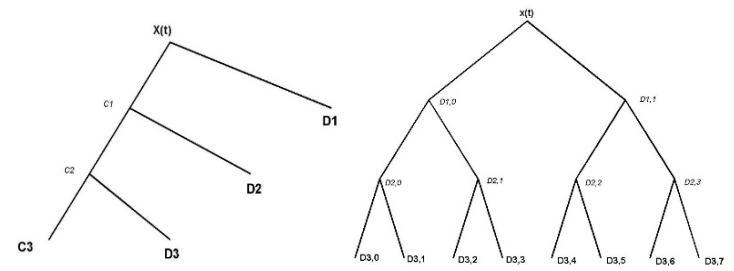

Figure 2. Wavelet trees: (a) DWT, (b) DWPT, 3 level decompositions.

For discrete wavelet packet transform, the coefficients $\tilde{X}$ at each resolution level $j$, given by Equation (6), can be thought as being the projection of time series $x$ in the directions defined by bases functions $\psi_{j, m, k}(t)$, where $m$ varies from 0 to $2^{j}-1$ indicating each one of the packets of the decomposition. Parameter $k$ is the position inside each packet.

$$
\tilde{X}(j, m, k)=\sum_{k} x(t) \psi_{j, m, k}(t)
$$

Here, the notation $\psi_{j, m, k}(t)$ is general and is used for indicating both scaling and wavelet function. In practice, the DWPT is performed also only with 
respect to the wavelet filters $h_{n}$ and $g_{n}$, and is represented at Fig. 2(b), where the blocks $D_{j, m}$ contain the coefficients $\tilde{X}_{j, m, k}$.

\section{EXPERIMENTAL TECHNIQUE}

The test section, presented in Fig. 3, is a $2000 \mathrm{~mm}$ long rectangular channel, with $146 \mathrm{~mm}$ height and $193 \mathrm{~mm}$ width. Air, drawn from the laboratory environment, is the working fluid, driven by a centrifugal blower. The blower velocity was controlled by a frequency inverter.

A single wire hot wire probe was placed at a fixed position to measure the reference velocity for the experiments. At the maximum velocity the Reynolds number, calculated for the cylinder diameter $(32.1 \mathrm{~mm})$ was $2.7 \times 10^{4}$. Measurements of the velocity in the wake of a single tube were performed using a constant temperature hot wire anemometer. A double hot wire probe was used, allowing the determination of both axial and transversal velocity components. Acquisition frequencies of $25 \mathrm{kHz}$ and $8 \mathrm{kHz}$ were used and low pass (anti-aliasing) filters of $10 \mathrm{kHz}$ and $3 \mathrm{kHz}$ were applied. At both experiments, the fan was turned on manually (about 1 second) after the start of the acquisition system. A constant acceleration for the flow velocity variation was set by adjusting the inverter for a linear frequency variation from 0 to $60 \mathrm{~Hz}$. At both experiments, the air velocity achieves its maximum value and becomes statistically stationary 4.5 seconds after the beginning of the dada acquisition.

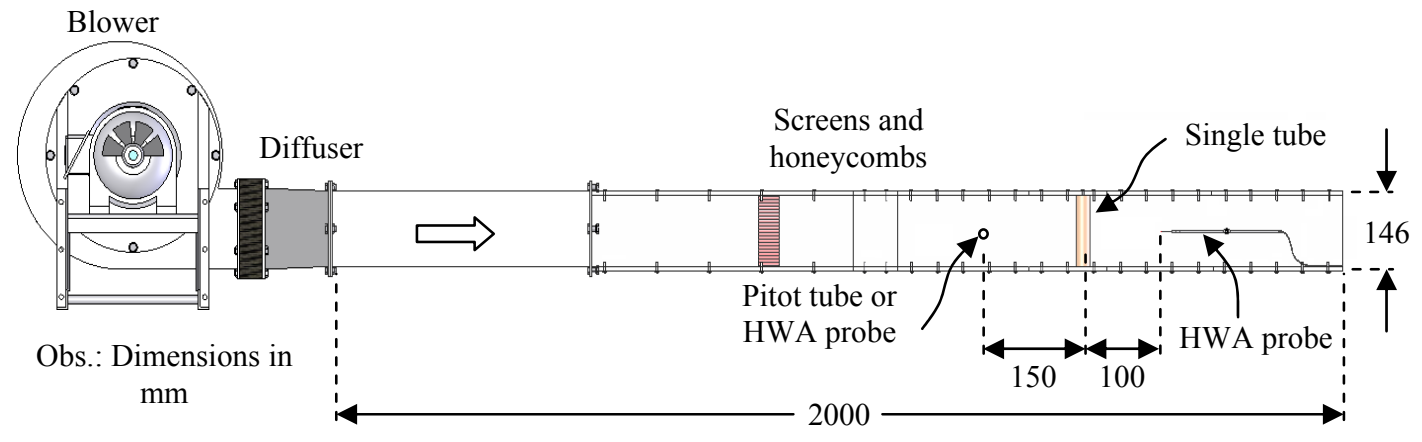

Figure 3. Schematic view of the test section.

\section{ANALYTICAL MODEL}

The wake of a single cylinder in both stationary and transient flows was used to confirm the ability of the CWT to allow the visualization of the energy distribution in the time-frequency domain.

For comparison with the transient signal acquired at a frequency of $8 \mathrm{kHz}\left(2^{16}\right.$ samples, 8.2 seconds), the evolution in the wake was simulated analytically by the function represented below:

$$
0 \leq t \leq 1.15 s \quad x(t)=0
$$

$1.15 s<t \leq 4.2 s \quad x(t)=\log (t) \sin \left(30 \pi t^{2}-58 \pi t\right)$

$$
4.2 s<t \leq 8.2 s \quad x(t)=\log 8 \sin (194 \pi t)
$$

Equation 8 is a sinusoidal function with a linear frequency modulation, known as chirp. Its instantaneous frequency is given by $(30 t-29) \mathrm{Hz}$. The chirp was chosen due to its resemblance to the real velocity signal at the transient period without the random component. Indeed, chirp is a continuously varying, in amplitude, frequency and mean value, signal. Wickersham et al. (2014), to highlight the performance of CWT in the analysis of flames, also used a similar signal. For Eq. 7, 8 and 9, the increment of $t$ was $1 / 8000$ seconds.

Figure 4 shows (a) the transient axial velocity and (b) the transient transversal velocity in the wake, $0.10 \mathrm{~m}$ downstream the cylinder, (c) reference velocity and (d) the analytically created signal. Steady state occurs at about 4.5 seconds after data acquisition started and about 3.7 seconds after the blower started.

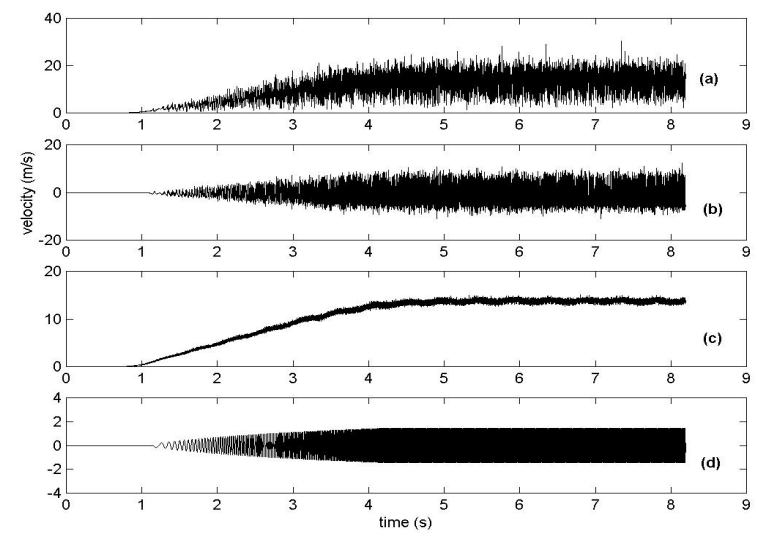

Figure 4. (a) Axial velocity in the wake of the cylinder, (b) transversal wake velocity, (c) reference velocity upstream the cylinder, (d) analytically created signal. 


\section{WAVELET ANALYSIS}

For discrete transforms (DWT and DWPT), the strategy for the current work was initially to explore all possible wavelet functions available on the MATLAB library and, according to preliminary results, to make a pre-selection for performing further analysis with nonstationary data. The four initially chosen were Haar, Meyer, and two orthonormal Daubechies functions, one with five null moments (Db5), and the other with 20 null moments (Db20). Preliminary analysis were done using both DWT and DWPT, of a typical stationary wake signal acquired at the wind tunnel at same conditions as the signal shown in Fig. 4 (sampling frequency of $25 \mathrm{kHz}$ ) (Indrusiak, 2004). The series resulting of reconstruction of the signals for some frequency intervals ( 0 to $6.1 \mathrm{~Hz}, 6.1$ to $12.2 \mathrm{~Hz}$ and 12.2 to $18.3 \mathrm{~Hz}$ ) were analyzed through Fourier spectra, showing that, for the signal investigated, the best frequency filtering ability of discrete wavelet transforms is obtained with $\mathrm{Db} 20$. The first three wavelets present high frequency components along with the expected frequency content, while in the latter high frequencies were completely eliminated. Indeed, spectra of the reconstructed approximation $(0$ to $6.1 \mathrm{~Hz}$ ) and details (6.1 to $12.2 \mathrm{~Hz}$ and 12.2 to $18.3 \mathrm{~Hz}$ ) of the signal, Fig. 5, show that the best frequency filter behavior was achieved by the use of Db20 wavelet as basis. Thus, for DWT and DWPT analysis, Db 20 was used.
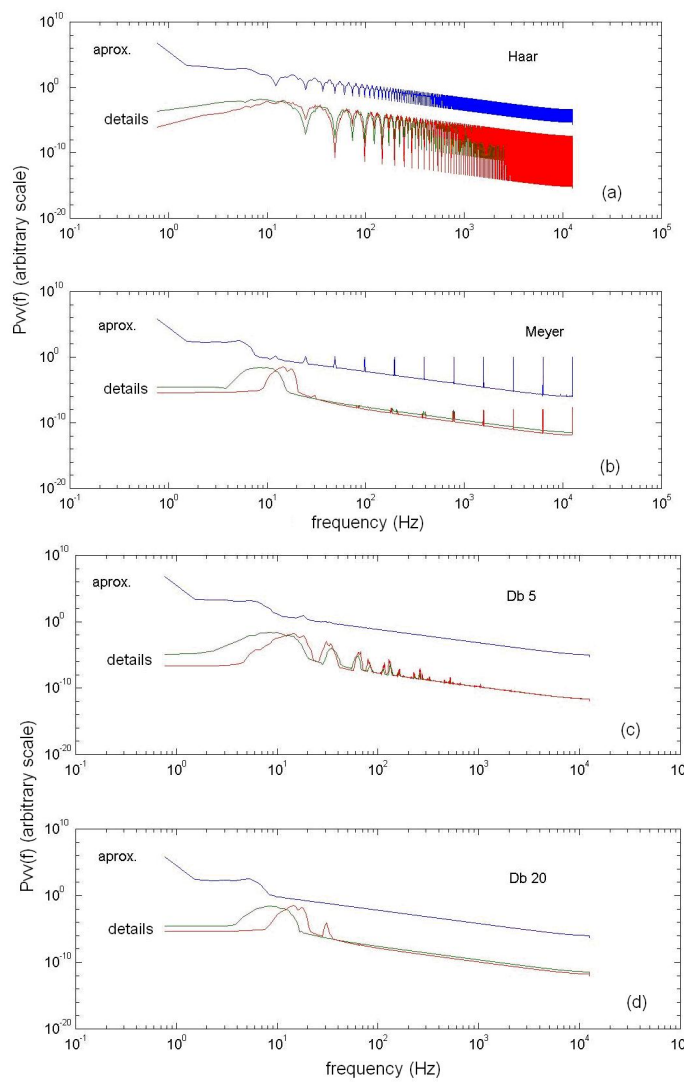

Figure 5. Power spectral density for the first three frequency intervals reconstructed from DWPT level $11((0$ to $6.1 \mathrm{~Hz}, 6.1$ to $12.2 \mathrm{~Hz}$ and 12.2 to 18.3

$\mathrm{Hz}$ ), using wavelet bases of (a) Haar, (b) Meyer, (c) Db5 and (d) Db20.

The Parseval identity ensures that discrete wavelet transforms for orthonormal basis conserve the energy of the signal. Figure 6 shows the energy spectra of stationary signal, calculated by Fourier, DWT and DWPT. Fourier spectrum was obtained using Welch (Welch, 1967) algorithm with a Hamming window function. Frequency interval was 6.1 Hz. For DWT, the first frequency interval was also $6.1 \mathrm{~Hz}$. Nevertheless, each subsequent interval has twice the frequency value of the previous one, due to the inherent characteristic of DWT. Indeed, also the peak related to vortex street was smoothed due to the very large interval $(97.6$ to $195.3 \mathrm{~Hz}$ ). For the spectrum obtained from DWPT frequency interval was $6.1 \mathrm{~Hz}$, equivalent to that of Fourier spectrum, for the first 128 intervals (up to $781 \mathrm{~Hz}$ ) and then due to computational reasons doubled at each 64 intervals, thus presenting a smoother aspect at higher frequencies.
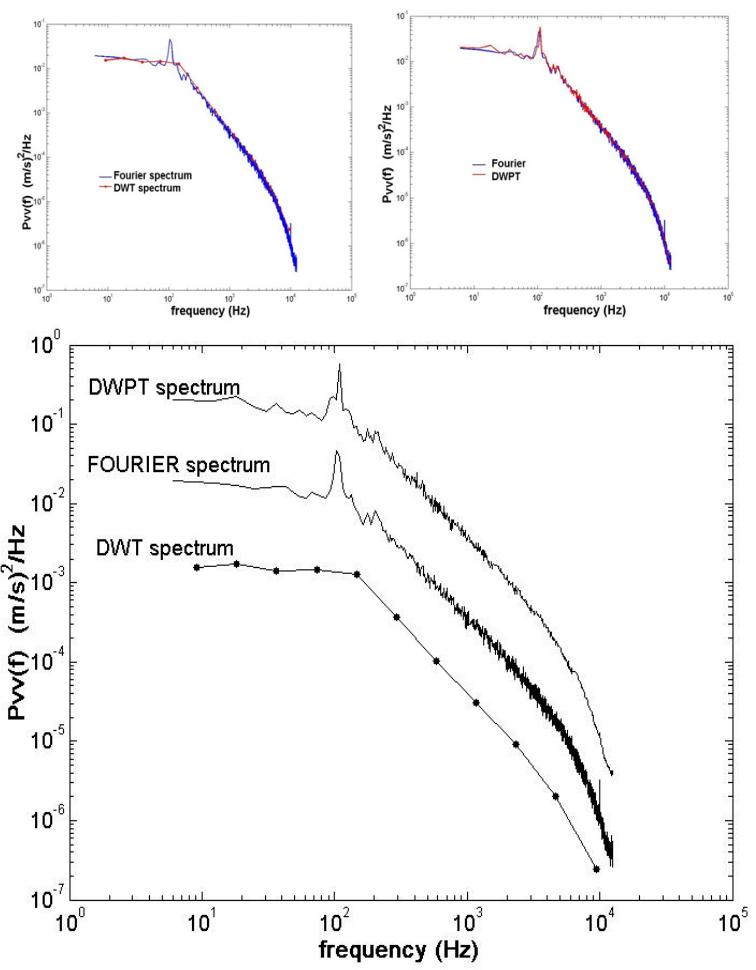

Figure 6. Power spectral density of the stationary wake signal, obtained by (a) Fourier transform and DWT, (b) Fourier transform and DWPT. In (c), DW and DWP spectra were respectively divided and multiplied by ten to allow better viewing.

Despite of the adjustments, spectra are coincident, as shown in Fig. 6(a) and 6(b). To allow better visualization wavelet and wavelet packet spectra were respectively divided and multiplied by 
ten in Fig. 6(c).

Another peculiarity of discrete wavelet transform, when assuming a periodic extension of the wavelet family in the analysis of finite dimensional data, is the appearance of spurious results arising from the discontinuities at both extremities of the finite experimental series. A usual procedure for Fourier transform is the application of a time window function (Bendat \& Piersol, 1986). A similar procedure may be applied to the signal prior to wavelet transformation. Another approach is to extend the series at the extremities considering any kind of data extrapolation (Kozakevicius and Schmidt, 2013). In addition, a confidence interval can be calculated to predict the region where results are reliable. In the present work the initial values of some series are null and also the acquisition time was extended beyond the interval of interest and thus no procedure was necessary.

Since the orthonormal wavelet transform preserves the energy of the signal, the distribution of energy in a time-frequency plane is represented by means of the squares of transform coefficients $\tilde{X}(a, b)$.of CWT. This is often called spectrogram or wavelet spectrum. For CWT, the Morlet function was adopted, following the tendency in the literature. Figure 7(a) shows the spectrogram of the analytical function (chirp) represented by Eqs. 7, 8 and 9 . Figure $7(\mathrm{~b})$ is the spectrogram of the signal acquired in the cylinder wake, at a sampling frequency of $8 \mathrm{kHz}$ and in Fig. 7(c), the background turbulence is shown. Wavelet spectrum of the chirp (Fig. 7(a)) shows a very regular increase of energy at increasing frequencies along time. It reproduces time evolution of the wake, Fig. 7(b), whose energy levels and frequencies increase along time during the transient time interval, presenting furthermore constant energy level and frequency interval at permanent part of the signal. This high-energy frequency interval related to the permanent wake spreads from 80 to $120 \mathrm{~Hz}$.

The continuous wavelet spectrum of the reference velocity (Fig. 7(c)) shows that, after the onset of the flow, at about 0.8 seconds, energy is distributed randomly over all frequencies, with levels increasing with time at transient part, especially at the interval from beginning to 1.5 seconds. The background of Fig. 7(b) is similar to that of Fig. 7(c), but with higher energy levels, denoting enhancement of energy of the flow at all scales due to the wake.

The comparison of Figs. 7(a-c) shows that the flow downstream the cylinder can be considered as a sum of a background of randomly distributed energy plus the very organized and high energy level (coherent) structure formed by the vortices transported by the flow. These observations can be corroborated by observing some details of the evolution of chirp and wake in the spectrograms for time interval from 3 to 3.3 seconds, and the background turbulence in the same interval in Fig. $8(\mathrm{a}-\mathrm{c})$, respectively.
As in time domain the analytical signal resembles the real series in the wake, also the spectrograms are similar. The main difference is that while energy distribution of the first one is regular, like the series itself, the actual series presents irregularities in the entire interval. This is because the wake is turbulent at very low Reynolds numbers. According to Blevins (1990) after a Reynolds number of 300 the wake is fully turbulent. This value corresponds, in the present case, to a flow velocity of about $0.15 \mathrm{~m} / \mathrm{s}$. The frequency of vortices in the wake fluctuates around the average frequency dictated by mean incident velocity and cylinder diameter. By means of Fourier analysis, one can obtain the average frequency and fluctuation of the wake remains completely hidden. Only by wavelet analysis, this feature is observed.
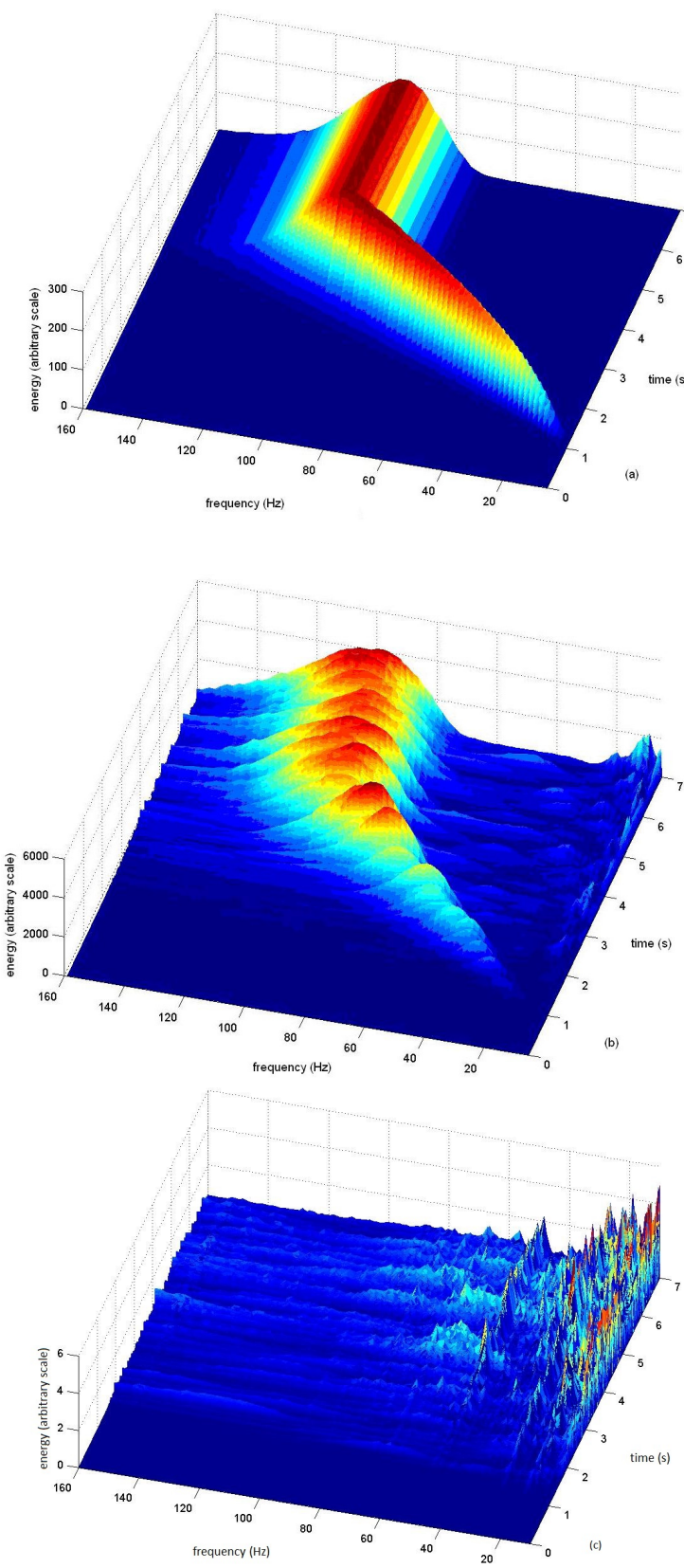


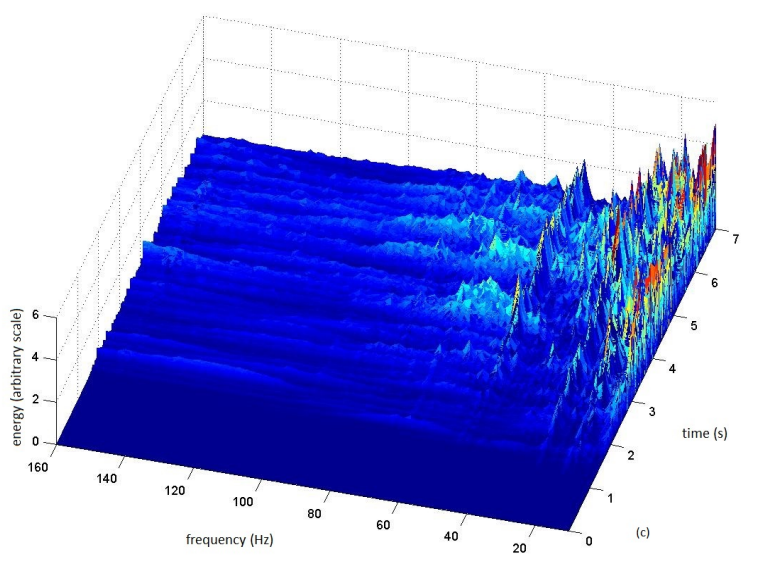

Figure 7. (a) Spectrogram of the analytical function (chirp) given by equations 7,8 and 9; (b) spectrogram of the signal acquired in the cylinder wake and (c) spectrogram of reference velocity. In (b) and (c) sampling frequency of $8 \mathrm{kHz}$.

The evolution of transient wake (acceleration period) is clearly visible and it is noticeable that both frequency and energy increase with time. Any cut in the graph, parallel to frequency-energy plane, shows the instantaneous power spectral distribution, while a cut parallel to time-energy plane shows the fluctuation of energy over time for the chosen frequency, as shown in the quantitative analysis of transient wake, using DWPT, by Indrusiak and Möller, 2011.
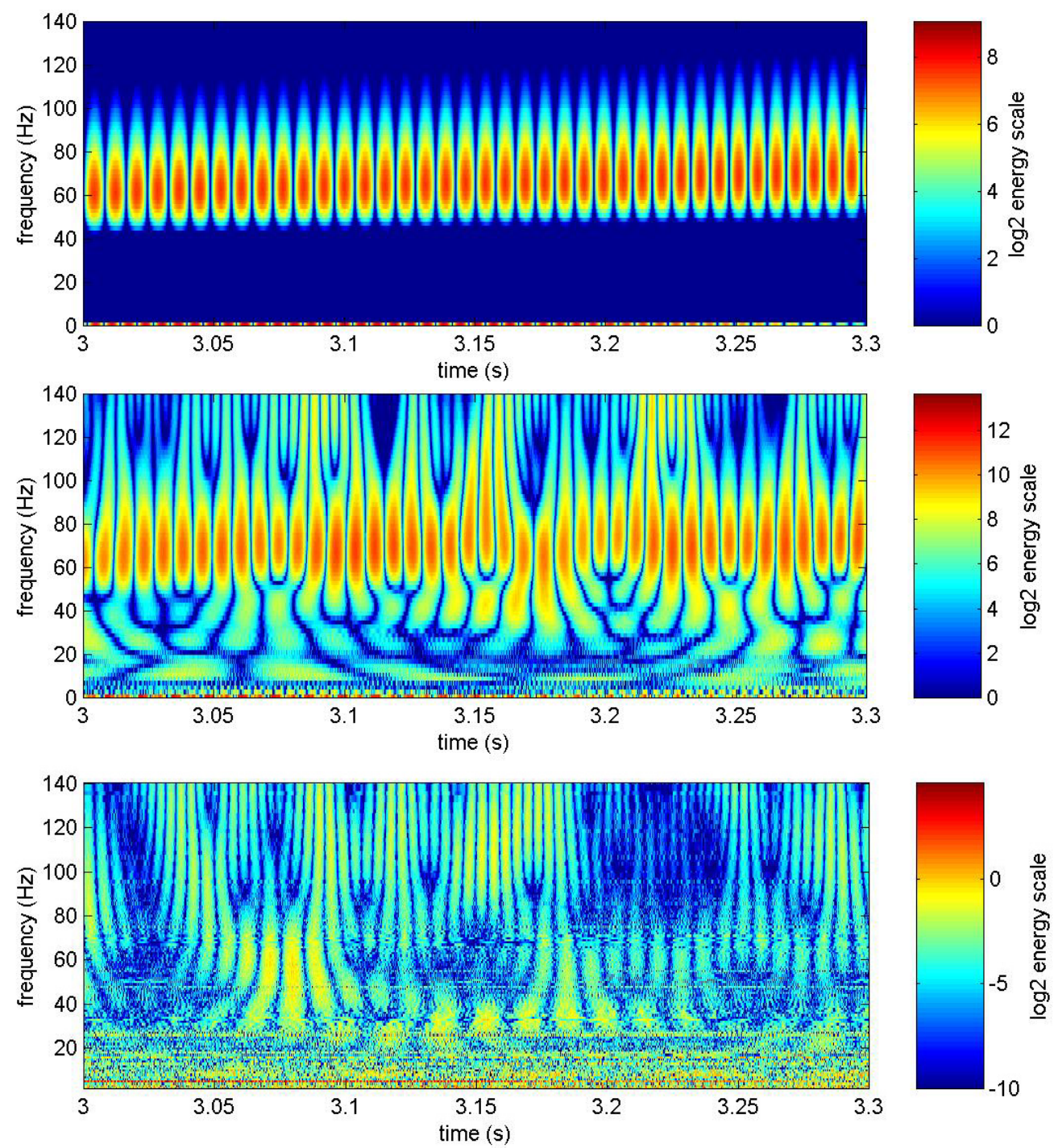

Figure 8. Details of Figs. 7 (a), (b) and (c).

\section{CONCLUSIONS}

The current work presents an overview of some relevant aspects for wavelet analysis when applied to stationary and nonstationary signals. The signal used was a transient wake past a circular cylinder. For discrete transforms, analyses performed over a stationary part of the signal show that DWT and 
DWPT performs like Fourier transform, preserving the energy content of the signal for each frequency band of the analysis. Continuous transform was applied to the whole signal, including transient part, and to a signal analytically constructed to have a similar behavior to the transient wake, with no broadband noise. Results show a very regular transient energy ridge for the analytical signal and a less regular ridge, surrounded by white noise energy, for the actual signal. For both, time-frequency evolution of the wake became clearly visible.

Some drawbacks of the choice of an appropriate wavelet function were presented. The ability of wavelet transforms to represent a given signal by splitting the whole range of signal frequencies (associated to different resolution scales and different characteristics) obtaining the entire time-frequency evolution is very important, since it allows a better understanding of random stationary and nonstationary processes.

The analyses performed over a transient signal, as well as over an analytically constructed signal, were designed ad hoc to show the applicability of the transforms. The wavelet function used for the CWT is a usual choice in the literature, but for discrete transforms, a non-exhaustive comparison was made between some available wavelet functions. The results show that the transforms are skillful to enhance the analysis of transient as well as stationary signals. In the chosen example, frequencies of the wake and their time location were known beforehand, but in phenomena where the frequencies of interest are not known or cannot be previously determined through Fourier analysis, the wavelet analysis can become a rather cumbersome process due to the search for more adequate wavelets and parameters. Therefore, wavelet transform cannot be considered simply an option to Fourier transform, but rather a complementary tool to be used to broaden understanding of the phenomenon or when traditional methods are unsatisfactory, as happens whenever nonstationary phenomena occur.

The ability of wavelet transform in filtering the signal analyzed in its various frequency ranges to visualize the evolution in each time interval, although not necessarily leading to the discovery of specific characteristics of flow, is very helpful for better understanding of phenomena studied. Wavelet transforms are versatile tools in experimental analysis because, while not requiring that the phenomenon is stationary, allow the time-frequency analysis of virtually any turbulent flow.

\section{ACKNOWLEDGEMENTS}

S.V. Möller is gratefully indebted to the CNPq National Council for Scientific and Technological Development, Ministry for Science, Technology and Innovation, Brazil, for the continuing financial support (BPP).
A. Kozakevicius acknowledges the grant FAPERGS - Foundation for Research Support in the State of Rio Grande do Sul, PG n.1873-25.51/13-0.

\section{REFERENCES}

Alam, M. M., Moriya, M., and Sakamoto, H., 2003, Aerodynamic Characteristics of Two Side-bySide Circular Cylinders and Application of Wavelet Analysis on the Switching Phenomenon, Journal of Fluids and Structures, Vol. 18, pp. 325-346.

Armstrong, W. J., 2011, Wavelet-Based Intensity Analysis of Mechanomyographic Signals During Single-Legged Stance Following Fatigue, Journal of Electromyography and Kinesiology, Vol. 21, No. 5, pp. 803-810.

Bendat, J. S., and Piersol, A. G., 1986, Random Data: Analysis and Measurement Procedures, $2^{\text {nd }}$ Ed.,Wiley-Interscience, New York.

Bendat J. S., and Piersol, A. G., 1993, Engineering Applications of Correlation and Spectral Analysis, Wiley-Interscience, New York.

Blevins, R. D., 1990, Flow-Induced Vibration, $2^{\text {nd }} E d$., Van Nostrand Reinhold, New York

Castillo, E., Morales, D. P., Botella, G., García, A., Parrilla, L., and Palma, A. J., 2013, Efficient Wavelet-Based ECG Processing for Single-Lead FHR Extraction, Digital Signal Processing, Vol. 23, pp. 1897-1909.

Comte-Bellot, G., 1965, Ecoulement turbulent entre deux parois parallèles, Publications Scientifiques e Techniques du Ministére de l'air, V. 419.

Crowley. P. M., 2007, A Guide to Wavelets for Economists, Journal of Economic Surveys, Vol. 21, pp. 207-267.

Daubechies, I., 1992, Ten Lectures on Wavelets, SIAM, Philadelphia, EUA.

Einstein, A., 1914, Eine Methode zur statistischen Verwertung von Beobachtungen Scheinbar Unregelmässig Quasiperiodisch verlaufenderVorgänge, The Collected Papers of Albert Einstein, Vol. 4., Princeton U. P., Princeton.

Farge, M., 1992, Wavelet Transforms and their Applications to Turbulence, Annual Review of Fluid Mechanics, Vol. 24, pp. 395-457.

Gabor, D., 1946, Theory of communication. Part 1: The analysis of information, Journal of the Institution of Electrical Engineers - Part III, Radio and Communication Engineering, Vol. 93, pp. 429441.

Galanti, B., and Tsinober, A., 2004, Is Turbulence Ergodic?, Physics Letters A, Vol. 330, pp. 173-180.

Hinze, J. O., 1975, Turbulence, McGraw-Hill, New York.

Hui, M. C. H., Larsen A., and Xiang, H. F., 2009, Wind Turbulence Characteristics Study at the Stonecutters Bridge site: Part I-Mean Wind and Turbulence Intensities, Journal of Wind Engineering 
and Industrial Aerodynamics, Vol. 97, pp. 22-36.

Hui, M. C. H., Larsen A., and Xiang, H. F., 2009, Wind Turbulence Characteristics Study at the Stonecutters Bridge site: Part II--Wind Power Spectra, Integral Length Scales and Coherences, Journal of Wind Engineering and Industrial Aerodynamics, Vol. 97, pp. 48-59.

Hussain, A. K. M. F., 1983, Coherent Structures - Reality and Myth, Physics of Fluids, Vol. 26, pp. 2816-2850.

Indrusiak, M. L. S., Goulart, J. V., Olinto C. R., and Möller, S. V., 2005, Wavelet Time Frequency Analysis of Accelerating and Decelerating Flows in a Tube Bank, Nuclear Engineering and Design, Vol. 235 , pp. $1875-1887$.

Indrusiak, M. L. S., and Möller, S. V., 2011, Wavelet Analysis of Unsteady Flows: Application on the Determination of the Strouhal Number of the Transient Wake Behind a Single Cylinder, Experimental Thermal and Fluid Science, Vol. 35, pp. 319-372.

Körner, T. W., 1988, Fourier Analysis, Cambridge University Press, New York.

Kozakevicius, A., and Schmidt, A. A., 2013, Wavelet Transform with Special Boundary Treatment for 1D Data, Computational \& Applied Mathematics, Vol. 32, No. 3, pp. 447-457.

Lawn, C. J., 1971, The Determination of the Rate of Dissipation in Turbulent Pipe Flow, Journal of Fluid Mechanics, Vol. 48, pp. 477-505.

Mallat, S. G., 1989, A Theory for Multiresolution Signal Decomposition: The Wavelet Representation, Academic Press, San Diego.

Mallat, S. G. 1998, A Wavelet Tour of Signal Processing, $2^{\text {nd }} E d$, Academic Press, London.

Meyer, Y., 1993, Wavelets: Algorithms and Applications, SIAM, New York.

Olinto, C. R., Indrusiak, M. L. S., Endres, L. A. M., and Möller, S. V., 2009, Experimental Study of the Characteristics of the Flow in the First Rows of Tube Banks, Nuclear Engineering and Design, Vol. 239, pp. 2022-2034.

Percival, D. B., and Walden, A. T., 2000, Wavelets Methods for Time Series Analysis, Cambridge University Press, Cambridge, UK.

Taylor, G. I., 1936, Correlation Measurements in a Turbulent Flow through a Pipe, Proceedings of the Royal Society of London. Series A, Mathematical and Physical Sciences, Vol. 157, pp. 537-546.

Strang, G., and Nguyen, T., 1996, Wavelets and Filter Banks, SIAM, 1996.

Govindan, S. M., Duraisamy, P., and Yuan, X., 2014, Adaptive Wavelet Shrinkage for Noise Robust Speaker Recognition, Digital Signal Processing, Vol. 33, pp. 180-190

Unser, M., and Blu, T., 2003, Mathematical Properties of the JPEG2000 Wavelet Filters, IEEE Transactions on Image Processing, Vol. 12, No. 9, pp. 1080-1090.

Velarde, G., Weyde, T., and Meredith, D., 2013,
An Approach to Melodic Segmentation and Classification based on Filtering with the Haar Wavelet, Journal of New Music Research, Vol. 42, No. 4, pp. 325-345.

Welch, P. D., 1967, The use of Fast Fourier Transform for the Estimation of Power Spectra: A Method Based on Time Averaging over Short, Modified Periodograms, IEEE Trans. Audio and Electroacoustics, Vol. AU-15, pp. 70-75.

Wickersham, A. J., Li, X., and Ma, L., 2014, Comparison of Fourier, Principal Component and Wavelet Analyses for High Speed Flame Measurements, Computer Physics Communications, Vol. 185, pp. 1237-1245.

Zdravkovich, M. M., and Stonebanks, K. L., 2000, Intrinsically Non-Uniform and Metastable Flow in a Behind Tube Arrays, Journal of Fluids and Structures, Vol. 4, pp. 305-319. 